Check for updates

King's Fund, London, UK

R.Murray@kingsfund.org.uk

Cite this as: BMJ 2021;373:n1555

http://dx.doi.org/10.1136/bmj.n1555

Published: 18 June 2021

\section{The NHS needs a comprehensive plan for recovery}

\author{
This system-wide challenge demands a system-wide response
}

\section{Richard Murray,}

Even before covid-19, the NHS was in need of some emergency treatment. In the 2019 general election ${ }^{1}$ the UK government promised 50000 more nurses, 6000 more general practitioners, and another 6000 other primary care professionals in addition to the 20000 already promised. The reason for these commitments was easy to see: widespread staff shortages and a slow but steady decline in many key markers of performance, whether in elective or accident and emergency care (where data are good) or in general practice, mental health, and community services (where they aren't).

Covid-19 then intervened, causing a massive disruption to services. At their lowest point, GP referrals to hospital fell by around $75 \%$ from the previous year, ${ }^{2}$ diagnostic tests by $70 \%,{ }^{3}$ emergency admissions by just under $40 \%,{ }^{4}$ and GP appointments by about a third. ${ }^{5}$ Elective activity is back to around $90 \%$ of pre-covid levels, ${ }^{6}$ although in any normal year a 10\% fall in activity would still be shocking.

The size of the hospital waiting list first dropped sharply as referrals dried up, before rising by 1.3 million as activity picked up again. By June 2021, even as covid-19 admissions to hospital once again begin to rise, signs of returning demand are appearing across the NHS. Attendances at major emergency departments hit their second highest level ever in May, ${ }^{4}$ and the volume of appointments with GPs has risen above pre-pandemic levels ${ }^{5}$ even without fully accounting for covid vaccinations.

\section{Long term solution}

Confronting these challenges will need a long term solution made up of four key elements. Firstly, although the visibility of the waiting list draws attention to the acute sector, solutions need to be about more than acute care. In general practice, despite the increases in activity, the number of GPs and practice nurses is not rising (measured by whole time equivalents) even if some progress is being made on the recruitment of other professions into primary care. ${ }^{7}$ Anecdotal reports of rising demand for mental health services are consistent with the effect of prolonged lockdowns and interruptions in care. ${ }^{8}$ This is a system-wide challenge and needs a system-wide response.

Secondly, we need long term planning. Some of the key resources-such as operating theatres and medical imaging scanners-don't get built overnight. Most critically of all, workforce shortages are so deep that they cannot be overcome quickly, and the service desperately needs a workforce plan that provides some relief to staff already exhausted by the past year while also setting out longer term staffing commitments and how they will be met. ${ }^{9}$
Thirdly, the plans will need funding. The government had set out spending totals for the NHS through this parliament, but these were all agreed before the pandemic and excluded essential budgets such as training, public health, and capital spending. To the pre-existing priorities from the NHS long term plan-many of which remain valid-we must now add dealing with growing backlogs of care, long covid, and an ongoing pandemic. Leaked estimates suggest that between £2bn and €1obn (€2bn-€12bn; $\$ 3 b n-\$ 14 b n)$ a year will be required to reduce elective waiting lists back to acceptable levels ${ }^{10}$ - the wide range underlines uncertainties over future trends in demand, not least from all the patients who should have been referred in 2020 but were not.

Fourthly, a proper conversation with the public is required. The NHS and the public will not be well served by overly optimistic commitments on recovery that cannot be delivered. This will mean living through a period when patients' overall expectations are not going to be met and making some tough prioritisation decisions until capacity and productivity recover.

Despite the tragedy of the pandemic, the health and care system enters this new era having learnt some valuable lessons from the response to covid-19. The digital experience has shown the NHS capable of largescale change at pace. The stronger relationships forged with both local government and parts of the voluntary sector also offer the chance to provide a more integrated service that should be better at tackling the longstanding inequalities that often need a system-wide approach.

If it all sounds too daunting, remember this has been done before. After years of decline, the NHS Plan in 2000 kicked off a decade of improving access and consistent and rapid increases in staff numbers-backed of course by a proper workforce plan and improving outcomes. If the government provides the resources, history shows the NHS can deliver.

Competing interests: I have read and understood BMJ policy on declaration of interests and have no interests to declare.

Provenance and peer review: Commissioned; not externally peer reviewed.

Conservative and Unionist Party. Manifesto. Get Brexit done: unleash Britain's potential. 2019. https://assets-global.websitefiles.com/5da42e2cae7ebd3f8bde353c/5dda924905da587992a064ba_Conservative\%202019\%20Manifesto.pdf

NHS England. Monthly outpatient referrals data. 2020-2021. https://www.england.nhs.uk/statistics/statistical-work-areas/outpatientreferrals/mrr-data/

NHS England. Monthly diagnostic waiting times and activity. 2020-2021. https://www.england.nhs.uk/statistics/statistical-work-areas/diagnosticswaiting-times-and-activity/monthly-diagnostics-waiting-times-and-activity/ 
4 NHS England. A\&E attendances and emergency admissions. 2020-2021. https://www.england.nhs.uk/statistics/statistical-work-areas/ae-waiting-times-and-activity/

5 NHS Digital. Appointments in general practice March 2021. https://digital.nhs.uk/data-and-information/publications/statistical/appointments-in-general-practice/march-2021

6 NHS England. NHS ahead of target in recovery of elective care, and mental health services back to pre-pandemic levels. 10 June 2021. https://www.england.nhs.uk/2021/06/nhs-ahead-of-targetin-recovery-of-elective-care-and-mental-health-services-back-to-pre-pandemic-levels/

7 NHS Digital. General practice workforce 31 March 2021. https://digital.nhs.uk/data-and-information/publications/statistical/general-and-personal-medical-services/31-march-2021

8 Discombe M. Mental health demand could rise by 40pc warns NHSE research. Health Service Journal2020 Dec 7. https://www.hsj.co.uk/mental-health/mental-health-demand-could-rise-by40pc-warns-nhse-research/7029085.article

9 House of Commons Health and Social Care Committee. Workforce burnout and resilience in the NHS and social care. 2021. https://publications.parliament.uk/pa/cm5802/cmselect/cmhealth/22/2205.htm

10 Campbell D, Duncan P. Fixing NHS waiting times could cost £40bn, leaked No 10 estimates show. Guardian 2021 June 10. https://www.theguardian.com/society/2021/jun/10/fixing-nhs-waitingtimes-could-cost-40bn-leaked-no-10-estimates-show 